\title{
Identifying Perioperative Anesthetic Factors associated with Postoperative Morbidity in Robot- Assisted vs. Open Pancreatoduodenectomy: A Cohort Study
}

Antoon van den Enden ( $\nabla$ t.vandenenden@hotmail.com )

Erasmus University Medical Center

Bas Groot Koerkamp

Erasmus University Medical Center

Maya Maranus-Vereen

Erasmus University Medical Center

Markus Klimek

Erasmus University Medical Center

\section{Research Article}

Keywords: Pancreatic surgery, robot-assisted surgery, pancreatoduodenectomy, perioperative guidance.

Posted Date: January 15th, 2021

DOI: https://doi.org/10.21203/rs.3.rs-145387/v1

License: (c) (1) This work is licensed under a Creative Commons Attribution 4.0 International License.

Read Full License 


\section{Abstract}

Background: Robot-assisted pancreatoduodenectomy (RAPD) is a challenging procedure for the perioperative anesthesiologist, e.g. because of prolonged pneumoperitoneum exposure and reversedTrendelenburg positioning. Purpose of this retrospective cohort study is to identify differences in perioperative anesthesia-related factors between RAPD and open pancreatoduodenectomy (OPD) and to determine perioperative anesthetic factors associated with major morbidity (Clavien Dindo $\geq$ III) after RAPD.

Methods: All consecutive patient undergoing pancreatoduodenectomy were retrospectively included during a two year inclusion period. Anesthesia charts were studied on fluid management details, rates of vasopressor administration and arterial blood gas results. All factors were compared between both surgical approaches. Within RAPD, factors were subsequently compared between patients with major (Clavien Dindo $\geq \mathrm{III}$ ) vs. without major postoperative morbidity and between procedures with high and low intraoperative blood loss. Perioperative factors associated with considerable postsurgical morbidity (Clavien Dindo $\geq$ III) were identified by constructing a logistic regression model.

Results: RAPD was associated with higher administration of intraoperative vasopressors $(9.5 \%$ of operative time vs. $0 \%$ in OPD, $p=0.005$ ) and a higher net intraoperative fluid balance ( 2497.6 vs. 1572.3 $\mathrm{ml}, \mathrm{p}<0.001)$. OPD patients more frequently and quantitatively received colloids compared to RAPD patients $(79.0 \%$ vs. $51.6 \%, p<0.001,1000.0$ vs. $500.0 \mathrm{ml}, \mathrm{p}<0.001)$. Intraoperative erythrocyte transfusion rate was $6.3 \%$ (4/64) for RAPD compared to $30.6 \%$ (19/62) for OPD ( $<<0.001)$. Colloid administration during surgery and hyperlactatemia after 12 hour postoperative admission were associated with major (Clavien Dindo $\geq \mathrm{III}$ ) morbidity after RAPD (OR 5.06 with 95\% Cl 1.49-17.20, p=0.009 and OR 3.18 with $95 \% \mathrm{Cl} 1.01-9.91, \mathrm{p}=0.047$, respectively).

Conclusions: RAPD is a challenging procedure for the perioperative anesthesiologist e.g. considering a higher perioperative demand for vasopressors. Perioperative anesthetic factors, including hemodynamics and fluid strategy might alter postoperative morbidity. However, current data is insufficient to make specific recommendations.

\section{Background}

The robot-assisted approach of pancreatoduodenectomy was first described in 2003 by Giulianotti et al. and has gained momentum as a minimally invasive technique for pancreatoduodenectomy surgery [1]. Robot-assisted pancreatoduodenectomy (RAPD) was already compared with conventional open pancreatoduodenectomy (OPD) for surgical outcomes by various cohort studies. Despite longer duration of surgery, RAPD is characterized by lower intraoperative blood loss with equal postoperative mortality rates and similar oncological outcome [2-4]. Although the surgical feasibility of RAPD is extensively reported, little is known about specific anesthesiologic concerns of RAPD and how perioperative anesthesia-related factors affect postoperative outcome after RAPD. 
The optimal intraoperative fluid regimen during abdominal (including pancreatic) surgery in relation to postsurgical morbidity is part of ongoing scientific debate. E.g., the 2018 RELIEF trial described an association between a more restrictive intraoperative net fluid balance (median 3.7 liters) and an increased rate of postsurgical kidney injury after major abdominal surgery [5]. Grant et al. however observed no differences in postoperative major morbidity rate after randomizing between either a net liberal $\left(12 \mathrm{ml} \mathrm{kg}^{-1} \mathrm{hr}^{-1}\right)$ vs. net restrictive $\left(6 \mathrm{ml} \mathrm{kg}^{-1} \mathrm{hr}^{-1}\right)$ intraoperative fluid balance for pancreatectomy surgery, both in a conventional and minimally-invasive approach [6]. RAPD presents several specific perioperative challenges. The patient is exposed to pneumoperitoneum and placed in reversed-Trendelenburg position during the most of the surgical time. This specific sequence has previously been described to alter central venous pressure, to increase blood carbon dioxide levels as well as to decrease cardiac output [7-9]. Compared to OPD, RAPD is expected to differ in perioperative vasopressor demands and levels of fluid administration.

The objective of this retrospective study was firstly to evaluate anesthesia-related differences of RAPD compared to OPD and secondly to identify independent anesthesia-related factors associated with postRAPD morbidity.

\section{Methods}

\section{Population and study characteristics}

The local Medical Ethics Committee approved the study with waiver for informed patient consent with reference MEC-2019-0090 (Medical Ethics Committee, Erasmus MC University Hospital, Rotterdam, the Netherlands). The study protocol is performed in accordance with the relevant guidelines.

All consecutive patients who underwent pancreatoduodenectomy (either RAPD or OPD) between January 1st 2017 and December 31st 2018 have been retrospectively included for analysis. RAPD has been performed in our tertiary referral center since January 2017. All procedures were undertaken by a dedicated team of two pancreatic surgeons or a pancreatic surgeon together with a pancreatic surgical fellow. RAPD was executed using the Da Vinci Model S robotic surgical device, which was later switched to the Model Xi (Intuitive Surgical Inc., Sunnyvale, CA, USA). OP was performed by or under direct supervision of three experienced consultant pancreatic surgeons. Different consultant anesthesiologists $(\mathrm{n}=39)$ were involved in both surgical modalities.

RAPD is characterized as full-robotic surgery, meaning both the resection and the reconstruction phase were conducted robotically-assisted. Patients were assigned to RAPD based on patient preference and availability of both the robot and the robotic surgical team. Patients were only excluded for RAPD in case of locally advanced pancreatic cancer. All patients were postoperatively admitted to a High Dependency Unit (HDU) or incidentally to an Intensive Care Unit (ICU). Protocols for postoperative management were identical for both units. Digital patient records were reviewed for patient demographics, intra- and postoperative management and postoperative outcome. 


\section{Demographic and baseline details}

Extracted baseline data included age, sex, body mass index (BMI) and medical history (comprising preoperative diabetes mellitus, any pulmonary, cardiac or vascular disease, any history of cerebrovascular accident (CVA) or transient ischemic attack (TIA), hypertension or prior kidney or liver failure). Besides, data were extracted on preoperative rates of any previous malignancy, (non-) abdominal surgery or neoadjuvant chemotherapy. Baseline comorbidity was graded according to American Society of Anesthesiologist's (ASA) score and Charlson Comorbidity Index (CCI) [10]. Laboratory results (including hemoglobin $(\mathrm{Hb})$, platelet count, estimated glomerular filtration rate (e-GFR) and levels of albumin, total bilirubin, creatinine and CA 19 - 9) were also extracted.

\section{Perioperative factors and postoperative outcome}

According to local protocol norepinephrine (NE) was routinely used as perioperative vasopressor adjunct. Anesthesia records were screened for NE doses on start and end of surgery as well as the frequency of an $\mathrm{NE}$ dosage exceeding $0.2 \mathrm{\mu g} \mathrm{kg}^{-1} \mathrm{~min}^{-1}$ (this dosage was chosen within our center as a cut-off for NEadministration via a central venous catheter instead of via a peripheral intravenous cannula). A central venous catheter was incidentally inserted based on preoperative anesthesiologist's assessment. The amount of times NE dosage exceeded $0.2 \mathrm{ug} \mathrm{kg}^{-1} \mathrm{~min}^{-1}$ was expressed as time span in minutes and as percentage of operating room time (time span between entering and leaving the operating theatre). Operative time was defined as the time interval between skin incision and wound closure.

Fluid balances, including the necessity of erythrocyte transfusion, were studies up to 24 hours postoperatively. Results of arterial blood gas (ABG) analyses (including blood pH, partial $\mathrm{CO}_{2}\left(\mathrm{pCO}_{2}\right)$ pressure, lactate and $\mathrm{Hb}$ count) were evaluated at 3 points in time: first available sample results during surgery, first available results upon HDU/ICU admission and first available results after a minimum of 12 hour HDU/ICU admission. The perioperative ABG results were also compared with the corresponding end tidal (et) $\mathrm{CO}_{2}$ level.

Time to detubation was defined as the time interval between wound closure and removal of the endotracheal tube. Pain scores, expressed as Numeric Rating Scale (NRS), were analyzed on postoperative days 1 and 3 . Standard postoperative analgesic regimen comprised paracetamol $(1000 \mathrm{mg}$ 4 times daily) and naproxen ( $750 \mathrm{mg} 3$ times daily). OPD was preferably performed under additional epidural analgesia (routinely using ropivacaine $0.2 \%$ combined with sufentanil $1 \mu \mathrm{g} \mathrm{ml}^{-1}$ ). For RAPD a patient controlled analgesia (PCA) device (morphine based) was used for postoperative analgesia. Once oral intake was possible again, epidural or PCA analgesia was if necessary converted to oral oxycodone.

Patient records were analyzed for the total hours of postoperative HDU/ICU stay and the rate of prolonged HDU/ICU admission (defined as exceeding 24 hours). Length of hospital stay was determined starting from the first day after surgery. Postoperative morbidity was graded on a 90-day postsurgical interval using the Clavien Dindo (CD) score as well as the Comprehensive Complication Index [11, 12]. A CD score $\geq$ III was defined as major postsurgical morbidity. Kidney failure was graded according to 
European Society of Anesthesiologists' (ESA) European Perioperative Clinical Outcome (EPCO) standards [13]. Mortality rates were calculated on postoperative days 30 and 90 .

\section{Data processing and statistical analysis}

Baseline and perioperative factors were primarily analyzed for RAPD and OPD totals. Factors were subsequently compared for RAPD patients with major postsurgical morbidity (CD $\geq$ III) vs. RAPD patients without major postsurgical morbidity. In order to determine whether anesthetic factors were influenced by intraoperative blood loss, factors ware also compared for RAPD patients with above vs. below median intraoperative blood loss (referred to as groups of high and low blood loss, respectively).

Normal distribution of numerical data was assessed using a combination of visual inspection of histograms and Q-Q plots and the Shapiro-Wilk test. Numerical data on averages were presented as mean (standard deviation, SD) or median (interquartile range, IQR) as appropriate. Categorical data were presented with frequencies and percentages. An independent sample T-test or Mann-Whitney U-test was performed in comparing numerical data, a $\chi^{2}$ or Fisher's exact test in categorical data. A logistic regression model was constructed using a backward stepwise approach to identify independent prognostic factors of major morbidity $(C D \geq I I I)$ after RAPD. Results herein were presented as odds ratio (OR) with corresponding 95\% confidence interval. Throughout the study two-tailed P-values of $<0.05$ were considered statistically significant. Statistical analysis was carried out using IBM SPSS Statistics (version 24.0, Armonk, NY, USA; IBM Corp.).

\section{Results}

During the inclusion period, 126 Consecutive patients underwent pancreatoduodenectomy ( $\mathrm{n}=64$ RAPD, $n=62$ OPD).

\section{RAPD vs. OPD}

No differences in age, BMI and baseline medical history could be demonstrated between RAPD and OPD patients (Table 1). Baseline hemoglobin level was lower for OPD (12.6 vs. $13.2 \mathrm{~g} \mathrm{dl}^{-1}, \mathrm{p}=0.049$ ). Operative time was $441.5 \mathrm{~min}$. in RAPD compared to $318.0 \mathrm{~min}$. in OPD ( $<<0.001$, Table 2). No RAPD procedures were converted to OPD. Net intraoperative fluid balance was higher in RAPD (2497.6 vs. $1572.3 \mathrm{ml}$ in OPD, $\mathrm{p}$ < 0.001). OPD patients more frequently and quantitatively received colloids compared to RAPD patients $(79.0 \%$ vs. $51.6 \%, p<0.001,1000.0$ vs. $500.0 \mathrm{ml}, \mathrm{p}<0.001)$. Average intraoperative blood loss was $250.0 \mathrm{ml}$ for RAPD compared to $1150.0 \mathrm{ml}$ for OPD $(p<0.001)$ with an intraoperative erythrocyte transfusion rate of $6.3 \%(4 / 64)$ for RAPD compared to $30.6 \%(19 / 62)$ for OPD $(p<0.001)$. For RAPD, Hb levels were higher during surgery, upon HDU/ICU admission and after a minimum of 12 hour HDU/ICU admission (12.6 vs. $11.9 \mathrm{~g} \mathrm{dl}^{-1}, \mathrm{p}=0.017,12.6$ vs. $11.3 \mathrm{~g} \mathrm{dl}^{-1}, \mathrm{p}<0.001$ and $12.1 \mathrm{vs.} 11.0 \mathrm{~g} \mathrm{dl}^{-}$ $1, \mathrm{p}<0.001$, respectively). 
Table 1

Demographic and baseline details for RAPD and OPD patients

\begin{tabular}{|c|c|c|c|c|c|c|}
\hline \multirow[t]{2}{*}{ Variables } & \multicolumn{3}{|c|}{ RAPD vs. OPD } & \multicolumn{3}{|c|}{ Post-RAPD morbidity } \\
\hline & $\begin{array}{l}\text { RAPD (n } \\
=64)\end{array}$ & $\begin{array}{l}\text { OPD }(n= \\
62)\end{array}$ & $P$ & $\begin{array}{l}\text { RAPD without } \\
\text { major morbidity } \\
(C D<I I I, n=36)\end{array}$ & $\begin{array}{l}\text { RAPD with } \\
\text { major } \\
\text { morbidity } \\
(C D \geq I I I, n= \\
28)\end{array}$ & $P$ \\
\hline Age (yr) & $67.8 \pm 9.8$ & $\begin{array}{l}65.5 \pm \\
10.1\end{array}$ & 0.188 & $66.8 \pm 9.7$ & $69.1 \pm 9.9$ & 0.340 \\
\hline $\operatorname{Sex}(M: F)$ & $1.0: 0.9$ & $1.0: 0.9$ & 0.424 & $1.0: 1.0$ & $1.0: 0.9$ & 0.806 \\
\hline $\mathrm{BMI}\left(\mathrm{kg} / \mathrm{m}^{2}\right)$ & $\begin{array}{l}25.0 \\
(18.9- \\
37.9)\end{array}$ & $\begin{array}{l}24.6 \\
(16.7- \\
40.7)^{2}\end{array}$ & 0.179 & $25.9 \pm 5.0$ & $26.0 \pm 3.3$ & 0.909 \\
\hline \multicolumn{7}{|l|}{ Medical history } \\
\hline Diabetes mellitus & $17(26.6)$ & $22(35.5)$ & 0.337 & $12(33.3)$ & $5(17.9)$ & 0.254 \\
\hline $\begin{array}{l}\text { Pulmonary } \\
\text { disease }\end{array}$ & $13(20.3)$ & $9(14.5)$ & 0.484 & $7(19.4)$ & $6(21.4)$ & 1.000 \\
\hline Cardiac disease & $17(26.6)$ & $15(24.4)$ & 0.839 & $9(25.0)$ & $8(28.6)$ & 0.782 \\
\hline Vascular disease & $2(3.1)$ & $5(8.1)$ & 0.269 & $1(2.8)$ & $1(3.6)$ & 1.000 \\
\hline CVA or TIA & $5(7.8)$ & $6(9.7)$ & 0.761 & $3(8.3)$ & $2(7.1)$ & 1.000 \\
\hline Hypertension & $28(43.8)$ & $25(40.3)$ & 0.721 & $11(30.6)$ & $17(60.7)$ & 0.023 \\
\hline $\begin{array}{l}\text { Kidney or liver } \\
\text { failure }\end{array}$ & $10(15.6)$ & $10(16.1)$ & 1.000 & $4(11.1)$ & $6(21.4)$ & 0.312 \\
\hline $\begin{array}{l}\text { Previous } \\
\text { malignancy }\end{array}$ & $19(29.7)$ & $18(29.0)$ & 1.000 & $9(25.0)$ & 10 (35.7) & 0.256 \\
\hline $\begin{array}{l}\text { Previous } \\
\text { abdominal surgery }\end{array}$ & $35(54.7)$ & $36(58.1)$ & 0.723 & $21(58.3)$ & $14(50.0)$ & 0.615 \\
\hline $\begin{array}{l}\text { Previous non- } \\
\text { abdominal surgery }\end{array}$ & 41 (64.1) & $43(69.4)$ & 0.574 & $22(61.1)$ & 19 (67.9) & 0.610 \\
\hline \multicolumn{7}{|l|}{ ASA } \\
\hline I & $8(12.5)$ & $4(6.9)^{4}$ & 0.543 & $6(16.7)$ & $2(7.1)$ & 0.460 \\
\hline$\|$ & $45(70.3)$ & $\begin{array}{l}41(70.3) \\
4\end{array}$ & & $25(69.4)$ & $20(71.4)$ & \\
\hline III & $11(17.2)$ & $\begin{array}{l}13(22.4) \\
4\end{array}$ & & $5(13.9)$ & $6(21.4)$ & \\
\hline
\end{tabular}




\begin{tabular}{|c|c|c|c|c|c|c|}
\hline \multirow[t]{2}{*}{ Variables } & \multicolumn{3}{|c|}{ RAPD vs. OPD } & \multicolumn{3}{|c|}{ Post-RAPD morbidity } \\
\hline & $\begin{array}{l}\text { RAPD }(n \\
=64)\end{array}$ & $\begin{array}{l}\text { OPD }(n= \\
62)\end{array}$ & $P$ & $\begin{array}{l}\text { RAPD without } \\
\text { major morbidity } \\
(C D<I I I, n=36)\end{array}$ & $\begin{array}{l}\text { RAPD with } \\
\text { major } \\
\text { morbidity } \\
(C D \geq I I I, n= \\
\text { (C8) }\end{array}$ & $P$ \\
\hline IV & 0 & 0 & & 0 & 0 & \\
\hline \multicolumn{7}{|l|}{$\begin{array}{l}\text { Charlson } \\
\text { Comorbidity Index }\end{array}$} \\
\hline Score & $5.4 \pm 2.0$ & $5.4 \pm 1.9$ & 0.900 & $5.1 \pm 1.9$ & $5.8 \pm 2.0$ & 0.161 \\
\hline Charlson $=0$ & 0 & 0 & & - & - & - \\
\hline Charlson $=1-3$ & $12(18.8)$ & $10(16.1)$ & 0.332 & $8(22.2)$ & $4(14.3)$ & 0.492 \\
\hline Charlson $=4-6$ & $34(53.1)$ & $35(56.5)$ & & $18(50.0)$ & $16(57.1)$ & \\
\hline Charlson = 7 & $18(28.1)$ & $17(27.4)$ & & $10(27.8)$ & $8(28.6)$ & \\
\hline $\begin{array}{l}\text { Preoperative } \\
\text { chemotherapy }\end{array}$ & $4(6.3)$ & $11(17.7)$ & 0.057 & $2(5.6)$ & $2(7.1)$ & 0.593 \\
\hline \multicolumn{7}{|l|}{$\begin{array}{l}\text { Baseline } \\
\text { laboratory }\end{array}$} \\
\hline $\begin{array}{l}\text { Hemoglobin level } \\
(\mathrm{mmol} / \mathrm{L})\end{array}$ & $8.2 \pm 0.9$ & $7.8 \pm 1.0$ & 0.049 & $8.0 \pm 0.9$ & $8.4 \pm 0.8$ & 0.116 \\
\hline $\begin{array}{l}\text { Platelet count }(x \\
\left.10^{9} / \mathrm{L}\right)\end{array}$ & $266 \pm 83$ & $293 \pm 78$ & 0.063 & $264(114-556)$ & $\begin{array}{l}236(135- \\
402)\end{array}$ & 0.365 \\
\hline $\begin{array}{l}\text { e-GFR }\left(\mathrm{ml} \cdot \mathrm{min}^{-}\right. \\
\left.1 \cdot 1.73^{-1} \mathrm{~m}^{2}\right)\end{array}$ & $\begin{array}{l}81.3 \pm \\
18.1\end{array}$ & $\begin{array}{l}82.9 \pm \\
17.5\end{array}$ & 0.610 & $84.4 \pm 17.2$ & $77.3 \pm 18.7$ & 0.123 \\
\hline $\begin{array}{l}\text { Albumin level } \\
(\mathrm{g} / \mathrm{L})\end{array}$ & $\begin{array}{l}42.1 \pm 7.2 \\
2\end{array}$ & $\begin{array}{l}41.8 \pm 4.6 \\
6\end{array}$ & 0.757 & $\begin{array}{l}41.0(24.0- \\
50.0)^{1}\end{array}$ & $\begin{array}{l}42.0(32.0- \\
75.0)^{1}\end{array}$ & 0.564 \\
\hline $\begin{array}{l}\text { Total bilirubin level } \\
\text { (umol/L) }\end{array}$ & $\begin{array}{l}11.5(3.0- \\
214.0)\end{array}$ & $\begin{array}{l}9.0(3.0- \\
208.0)^{1}\end{array}$ & 0.218 & $12.5(3.0-214.0)$ & $\begin{array}{l}10.5(5.0- \\
51.0)\end{array}$ & 0.542 \\
\hline $\begin{array}{l}\text { Creatinine level } \\
\text { (umol/L) }\end{array}$ & $\begin{array}{l}77.0 \pm \\
23.7\end{array}$ & $\begin{array}{l}75.4 \pm \\
18.8\end{array}$ & 0.675 & $73.5 \pm 17.3$ & $81.4 \pm 29.7$ & 0.216 \\
\hline Ca $19-9$ (kU/L) & $\begin{array}{l}33.0(1.0- \\
5146.0)\end{array}$ & $\begin{array}{l}29.0(1.0- \\
7408.0)^{5}\end{array}$ & 0.880 & $\begin{array}{l}35.0(1.0- \\
5146.0)\end{array}$ & $\begin{array}{l}33.0(1.0- \\
1908.0)\end{array}$ & 0.946 \\
\hline
\end{tabular}




\begin{tabular}{|c|c|c|c|c|c|c|}
\hline \multirow[t]{2}{*}{ Variables } & \multicolumn{3}{|c|}{ RAPD vs. OPD } & \multicolumn{3}{|c|}{ Post-RAPD morbidity } \\
\hline & $\begin{array}{l}\text { RAPD (n } \\
=64)\end{array}$ & $\begin{array}{l}\text { OPD }(n= \\
62)\end{array}$ & $P$ & $\begin{array}{l}\text { RAPD without } \\
\text { major morbidity } \\
(C D<I I I, n=36)\end{array}$ & $\begin{array}{l}\text { RAPD with } \\
\text { major } \\
\text { morbidity } \\
(\mathrm{CD} \geq \mathrm{III}, \mathrm{n}= \\
\text { 28) }\end{array}$ & $P$ \\
\hline \multicolumn{7}{|c|}{$\begin{array}{l}\text { Values are presented as number (proportion) or depending on normality distribution of cases as } \\
\text { mean } \pm \text { SD or median (interquartile range). } \mathrm{X}^{\mathrm{n}} \text { where } \mathrm{n} \text { represents the number of missing cases. ASA } \\
\text { American Society of Anesthesiologists Classification; BMI, Body Mass Index; CD, Clavien Dindo; CVA } \\
\text { Cerebro Vascular Accident; -GFR, Estimated Glomerular Filtration Rate; OPD, Open } \\
\text { Pancreatoduodenectomy; RAPD, Robot-Assisted Pancreatoduodenectomy; TIA, Transient Ischemic } \\
\text { Attack. }\end{array}$} \\
\hline
\end{tabular}


Table 2

Perioperative anesthesia-related factors, RAPD vs. OPD

\begin{tabular}{|c|c|c|c|}
\hline \multirow[t]{2}{*}{ Variables } & \multicolumn{3}{|l|}{ RAPD vs. OPD } \\
\hline & RAPD (n = 64) & OPD $(n=62)$ & $P$ \\
\hline Operating room time (min) & $513.5(377.0-836.0)$ & $392.5(240.0-802.0)$ & $\dot{0} 001$ \\
\hline Operative time (min) & $441.5(326.0-756.0)$ & $318.0(188.0-753.0)$ & $<.001$ \\
\hline \multicolumn{4}{|l|}{ Intraoperative fluid balance } \\
\hline Net positive fluid balance (ml) & $\begin{array}{l}2497.6(544.0- \\
5535.0)^{1}\end{array}$ & $\begin{array}{l}1572.3(50.0- \\
25925.2)^{3}\end{array}$ & $\dot{0.001}$ \\
\hline Crystalloid dose (ml) & $\begin{array}{l}2100.0(51.0- \\
5137.0)^{1}\end{array}$ & $\begin{array}{l}1896.0(0.0-8337.4) \\
3\end{array}$ & 0.069 \\
\hline Colloid dose (ml) & $\begin{array}{l}500.0(0.0-2000.0) \\
1\end{array}$ & $\begin{array}{l}1000.0(0.0-5700.0) \\
3\end{array}$ & $\begin{array}{l}<.001 \\
0.001\end{array}$ \\
\hline Colloid administration & $33.0(51.6)^{1}$ & $49.0(79.0)^{3}$ & $\stackrel{<}{<.001}$ \\
\hline Blood loss (ml) & $250.0(0.0-2500.0)$ & $\begin{array}{l}1150.0(0.0- \\
11585.0)^{3}\end{array}$ & $\dot{0.001}$ \\
\hline Intraoperative erythrocyte transfusion & $4(6.3)^{1}$ & $19(30.6)$ & $\dot{0} 001$ \\
\hline \multicolumn{4}{|l|}{ NE regimen } \\
\hline NE dose on surgery's start (ug $\left.\cdot \mathrm{kg}^{-1} \cdot \mathrm{min}^{-1}\right)$ & $0.05(0.00-0.20)^{2}$ & $0.03(0.00-0.72)^{2}$ & 0.021 \\
\hline NE dose on surgery's end (ug. $\left.\mathrm{kg}^{-1} \cdot \mathrm{min}^{-1}\right)$ & $0.06(0.00-0.38)^{1}$ & $0.06(0.00-0.80)$ & 0.821 \\
\hline $\mathrm{NE}$ dose $>0.2 \mathrm{ug} \cdot \mathrm{kg}^{-1} \cdot \mathrm{min}^{-1}(\mathrm{~min})$ & $1(0-5)^{1}$ & $0(0-3)^{1}$ & 0.005 \\
\hline Time span NE dose $>0.2 \mathrm{ug} \cdot \mathrm{kg}^{-1} \cdot \mathrm{min}^{-1}(\mathrm{~min})$ & $4.1(0.0-610.0)^{1}$ & $0.0(0.0-393.0)^{1}$ & 0.002 \\
\hline Operative time NE dose $>\mathrm{ug} \cdot \mathrm{kg}^{-1} \cdot \mathrm{min}^{-1}(\%)$ & $9.5(0.0-96.2)^{1}$ & $0.0(0.0-56.5)^{1}$ & 0.005 \\
\hline \multicolumn{4}{|l|}{ First arterial BGA during surgery } \\
\hline Blood pH & $7.32 \pm 0.06^{7}$ & $7.35 \pm .0 .06^{2}$ & 0.021 \\
\hline Partial $\mathrm{CO}_{2}$ pressure $(\mathrm{kPa})$ & $6.2(4.3-24.2)^{7}$ & $5.6(4.5-7.1)^{2}$ & $<.001$ \\
\hline Corresponding end-tidal $\mathrm{CO}_{2}(\mathrm{kPa})$ & $5.0 \pm 0.6^{15}$ & $4.7 \pm 0.3^{8}$ & 0.001 \\
\hline
\end{tabular}




\begin{tabular}{|c|c|c|c|}
\hline \multirow[t]{2}{*}{ Variables } & \multicolumn{3}{|l|}{ RAPD vs. OPD } \\
\hline & RAPD $(n=64)$ & OPD $(n=62)$ & $P$ \\
\hline Lactate level (mmol/L) & $0.7(0.3-1.9)^{7}$ & $0.7(0.3-2.7)^{2}$ & 0.884 \\
\hline Hemoglobin count (mmol/L) & $7.8 \pm 0.8^{7}$ & $7.4 \pm 1.0^{2}$ & 0.017 \\
\hline \multicolumn{4}{|c|}{ First arterial BGA upon HCU/IDU admission } \\
\hline Blood pH & $7.33 \pm 0.04^{1}$ & $7.35 \pm 0.05$ & 0.013 \\
\hline Partial $\mathrm{CO}_{2}$ pressure $(\mathrm{kPa})$ & $5.8(4.7-20.1)^{1}$ & $5.5(4.0-6.7)$ & 0.002 \\
\hline Lactate level (mmol/L) & $1.3(0.1-6.3)^{1}$ & $1.3(0.4-11.7)$ & 0.517 \\
\hline Hemoglobin count (mmol/L) & $7.8 \pm 0.9^{1}$ & $7.0 \pm 1.0$ & $<.001$ \\
\hline \multicolumn{4}{|c|}{$\begin{array}{l}\text { First arterial BGA after } \geq 12 \text { hours HCU/IDU } \\
\text { admission }\end{array}$} \\
\hline Blood pH & $7.39(7.29-7.48)^{4}$ & $7.39(7.23-7.47)^{1}$ & 0.981 \\
\hline Partial $\mathrm{CO}_{2}$ pressure $(\mathrm{kPa})$ & $5.8(4.7-7.0)^{4}$ & $5.5(3.8-7.9)^{1}$ & 0.019 \\
\hline Lactate level (mmol/L) & $1.1(0.5-2.9)^{4}$ & $1.0(0.4-5.6)^{1}$ & 0.803 \\
\hline Hemoglobin count (mmol/L) & $7.5 \pm 0.9^{4}$ & $6.8 \pm 1.0^{1}$ & $<.001$ \\
\hline \multicolumn{4}{|c|}{$\begin{array}{l}\text { Values are presented as number (proportion) or depending on normality distribution of cases as } \\
\text { mean } \pm \text { SD or median (interquartile range). } X^{n} \text { where } n \text { represents the number of missing cases. BGA, } \\
\text { Blood Gas Analysis; HDU, High Dependency Unit; ICU, Intensive Care Unit; NE, Norepinephrine; OPD, } \\
\text { Open Pancreatoduodenectomy; RAPD, Robot Assisted Pancreatoduodenectomy. }\end{array}$} \\
\hline
\end{tabular}

On average, NE was administered in a higher dose at procedure's start in RAPD ( $0.05 \mathrm{vs} .0 .03 \mu \mathrm{g} \mathrm{kg}^{-1}$ $\min ^{-1}$ in OPD, $p=0.021$ ). During the intraoperative course of RAPD, NE dosage exceeded $0.2 \mu \mathrm{g} \mathrm{kg}^{-1}$ $\min ^{-1}$ more frequently compared to OP with a net time span of $48 \mathrm{vs.} 0 \mathrm{~min}(p=0.002)$ and $9.5 \mathrm{vs} .0$ percent of operating room time $(p=0.005)$. RAPD was characterized by lower average blood $\mathrm{pH}$ during surgery (7.32 vs. 7.35 in OPD, $\mathrm{p}=0.021)$ with high $\mathrm{pCO}_{2}$ pressures and corresponding et $\mathrm{CO}_{2}$ levels $(6.2$ vs. $5.6 \mathrm{kPa}$ in OPD, $p<0.001$ and 5.0 vs. $4.7 \mathrm{kPa}$ in OPD, $p=0.001$, respectively). A similar trend is observed in RAPD patients upon HDU/ICU arrival (arterial blood pH 7.33 vs. 7.35 in OPD, $p=0.013$ and $\mathrm{pCO}_{2} 5.8$ vs. $5.5 \mathrm{kPa}$ in OPD, $\mathrm{p}=0.002$, Table 2).

No differences were observed in major postoperative morbidity rate $(C D \geq I I I)$ between both surgical modalities $(28 / 64,43.8 \%$ in RAPD vs. $33 / 62,53.2 \%$ in OPD, $p=0.373$, Table 3$)$. An average Comprehensive Complication Index of 32.7 was observed in RAPD vs. 49.9 in OPD ( $p=0.012)$. Rates of postoperative acute kidney injury were $9 / 64(14.5 \%)$ for RAPD vs. $6 / 92(9.7 \%)$ for OPD $(p=0.583)$. Six 
patients entered the procedure with pre-existing renal impairment (e-GFR $<60 \mathrm{ml} \min -1, \mathrm{n}=3$ in RAPD and $\mathrm{n}=3$ in OPD). No further deterioration of kidney injury was observed in any of these patients. Average pain score on postoperative day 1 was 3 for RAPD compared to 1 for OPD $(p<0.001)$. On postoperative day 3 , average pain score was 2 for both RAPD and OPD $(p=0.894)$.

Table 3

Postoperative outcome after RAPD vs. OPD

\begin{tabular}{|c|c|c|c|}
\hline Variables & RAPD $(n=64)$ & OPD $(n=62)$ & $P$ \\
\hline Time to detubation (min) & $32.0(0.0-931.0)^{11}$ & $21.0(1.0-21714.0)^{9}$ & 0.381 \\
\hline Stay HCU/IDU (hours) & $19.5(14.6-97.4)$ & $21.6(15.6-478.4)^{1}$ & $<0.001$ \\
\hline Prolonged HDU/ICU admission & $5(7.8)$ & $12(19.7)^{1}$ & 0.069 \\
\hline Hospital stay (days) & $11.5(4.0-61.0)$ & $14.5(6.0-200.0)^{\circ}$ & 0.277 \\
\hline Comprehensive Complication Index & $32.7(0.0-100.0)$ & $49.9(8.7-100.0)$ & 0.012 \\
\hline \multicolumn{4}{|l|}{ CD morbidity rates } \\
\hline Grade III & $20(31.1)$ & $23(37.1)$ & 0.574 \\
\hline Grade IIIA & $15(23.4)$ & $14(22.6)$ & 1.000 \\
\hline Grade IIIB & $5(7.8)$ & $9(14.5)$ & 0.268 \\
\hline Grade IV & $8(12.5)$ & $10(16.1)$ & 0.617 \\
\hline Grade IVA & $3(4.7)$ & $4(6.5)$ & 0.715 \\
\hline Grade IVB & $5(7.8)$ & $6(9.7)$ & 0.761 \\
\hline$\Rightarrow>$ Grade III & $28(43.8)$ & $33(53.2)$ & 0.420 \\
\hline Acute Kidney failure & $9(14.5)$ & $6(9.4)$ & 0.583 \\
\hline 30-day mortality & $1(1.6)$ & $2(3.2)$ & 0.616 \\
\hline 90-day mortality & $5(7.8)$ & $4(6.5)$ & 1.000 \\
\hline NRS Postoperative day 1 & $3(0-7)^{8}$ & $1(0-7)^{3}$ & $<0.001$ \\
\hline NRS Postoperative day 3 & $2(0-7)^{9}$ & $2(0-5)^{16}$ & 0.894 \\
\hline \multicolumn{4}{|c|}{$\begin{array}{l}\text { Values are presented as number (proportion) or depending on normality distribution of cases as } \\
\text { mean } \pm \text { SD or median (interquartile range). } X^{n} \text { : where represents the number of missing cases. CD, } \\
\text { Clavien Dindo; HDU, High Dependency Unit; ICU, Intensive Care Unit; NRS, Numeric Rating Scale; OPD, } \\
\text { Open Pancreatoduodenectomy; RAPD, Robot Assisted Pancreatoduodenectomy. }\end{array}$} \\
\hline
\end{tabular}


A higher rate of baseline hypertension was observed in the RAPD group with major postoperative morbidity $(17 / 28,60.7 \%$ vs. $11 / 36,30.6 \%$ for the RAPD group without major morbidity, $p=0.023$, Table 1$)$. Intraoperative colloid administration and blood loss were higher in the RAPD group with major postoperative morbidity (500.0 vs. $0.0 \mathrm{ml}, p=0.002$ and 350.0 vs. $200.0 \mathrm{ml}, p=0.047$, respectively, Table 4). Average NE dose was higher at the end of surgery for the RAPD group with major postoperative morbidity ( 0.09 vs. $0.04 \mathrm{\mu g} \mathrm{kg}^{-1} \mathrm{~min}^{-1}$ for the RAPD group without major postoperative morbidity, $\mathrm{p}=$ 0.726). Upon HDU/ICU admission, lower arterial blood $\mathrm{pH}$ as well as higher lactate levels were observed in the RAPD group with major postoperative morbidity (7.32 vs. $7.34, p=0.017$ and 1.7 vs $1.3 \mathrm{mmol}^{-1}$, in the RAPD group without major postoperative morbidity, respectively). A similar trend was observed after a minimum of 12 hour HDU/ICU admission (7.37 vs. 7.39, $p=0.016$ and 1.4 vs. $0.9 \mathrm{mmol} \mathrm{I}^{-1}$, respectively). Hospital stay was doubled in the RAPD group with major postoperative morbidity compared to the RAPD group without major postoperative morbidity (18.0 vs. 7.0 days, $p<0.001$, Table 5 ). Within the 90 -day inclusion period, $n=2$ (RAPD) patients deceased due to early recurrence of malignant disease. 
Table 4

Perioperative anesthetic factors in RAPD

\begin{tabular}{|c|c|c|c|c|c|c|c|}
\hline \multirow[t]{3}{*}{ Variables } & \multirow[t]{3}{*}{$\begin{array}{l}\text { RAPD }(n \\
=64)\end{array}$} & \multicolumn{3}{|c|}{ Post-RAPD morbidity } & \multicolumn{3}{|c|}{$\begin{array}{l}\text { Intraoperative blood loss } \\
\text { (RAPD) }\end{array}$} \\
\hline & & \multirow{2}{*}{$\begin{array}{l}\text { RAPD } \\
\text { with } \\
\text { major } \\
\text { morbidity } \\
(C D \geq I I I, \\
n=28)\end{array}$} & \multirow{2}{*}{\multicolumn{2}{|c|}{$\begin{array}{l}\text { RAPD } \\
\text { without } \\
\text { major } \\
\text { morbidity } \\
(C D<\text { III, } n \\
=36)\end{array}$}} & \multirow[t]{2}{*}{$\begin{array}{l}\text { High } \\
(\geq \\
250 \mathrm{ml}, \\
\mathrm{n}=33)\end{array}$} & \multirow[t]{2}{*}{$\begin{array}{l}\text { Low } \\
(< \\
250 \mathrm{ml}, \\
\mathrm{n}=31)\end{array}$} & \multirow[t]{2}{*}{$P$} \\
\hline & & & & & & & \\
\hline $\begin{array}{l}\text { Operating room } \\
\text { time }\end{array}$ & $\begin{array}{l}513.5 \\
(377.0- \\
836.0)\end{array}$ & $\begin{array}{l}527.5 \\
(397.0- \\
749.0)\end{array}$ & $\begin{array}{l}513.5 \\
(377.0- \\
836.0)\end{array}$ & 0.690 & $\begin{array}{l}580.0 \\
(397.0- \\
750.0)\end{array}$ & $\begin{array}{l}478.0 \\
(377.0- \\
836.0)\end{array}$ & 0.001 \\
\hline Operative time & $\begin{array}{l}441.5 \\
(326.0- \\
756.0)\end{array}$ & $\begin{array}{l}463.5 \\
(353.0- \\
691.0)\end{array}$ & $\begin{array}{l}441.5 \\
(326.0- \\
756.0)\end{array}$ & 0.671 & $\begin{array}{l}516.0 \\
(353.0- \\
691.0)\end{array}$ & $\begin{array}{l}410.0 \\
(326.0- \\
756.0)\end{array}$ & 0.001 \\
\hline \multicolumn{8}{|l|}{$\begin{array}{l}\text { Intraoperative fluid } \\
\text { balance }\end{array}$} \\
\hline $\begin{array}{l}\text { Net positive fluid } \\
\text { balance (ml) }\end{array}$ & $\begin{array}{l}2497.6 \\
(544.0- \\
5535.0) \\
1\end{array}$ & $\begin{array}{l}2777.1 \pm \\
1046.9^{1}\end{array}$ & $\begin{array}{l}2688.0 \pm \\
806.3\end{array}$ & 0.704 & $\begin{array}{l}3057.0 \\
(544.0- \\
5535.0) \\
1\end{array}$ & $\begin{array}{l}2288.9 \\
(1525.6- \\
4346.9)\end{array}$ & 0.012 \\
\hline $\begin{array}{l}\text { Crystalloid dose } \\
(\mathrm{ml})\end{array}$ & $\begin{array}{l}2100.0 \\
(51.0- \\
5137.0) \\
1\end{array}$ & $\begin{array}{l}2267.4 \pm \\
1110.4^{1}\end{array}$ & $\begin{array}{l}2294.4 \pm \\
702.2\end{array}$ & 0.906 & $\begin{array}{l}2478.7 \\
\pm \\
1048.7 \\
1\end{array}$ & $\begin{array}{l}2080.6 \\
6 \pm 51.6\end{array}$ & 0.076 \\
\hline Colloid dose (ml) & $\begin{array}{l}500.0 \\
(0.0- \\
2000.0) \\
1\end{array}$ & $\begin{array}{l}500.0 \\
(0.0- \\
2000.0)^{1}\end{array}$ & $\begin{array}{l}0.0(1.0- \\
1000.0)\end{array}$ & 0.002 & $\begin{array}{l}500.0 \\
(0.0- \\
2000.0) \\
1\end{array}$ & $\begin{array}{l}0.0(0.0- \\
1250.0)\end{array}$ & $\begin{array}{l}< \\
0.001\end{array}$ \\
\hline $\begin{array}{l}\text { Colloid } \\
\text { administration }\end{array}$ & $\begin{array}{l}33.0 \\
(51.6)^{1}\end{array}$ & $\begin{array}{l}20(71.4) \\
1\end{array}$ & $13(36.1)$ & 0.005 & $\begin{array}{l}25 \\
(75.8)^{1}\end{array}$ & $8(25.8)$ & $\dot{0.001}$ \\
\hline Blood loss (ml) & $\begin{array}{l}250.0 \\
(0.0- \\
2500.0)\end{array}$ & $\begin{array}{l}350.0 \\
(0.0- \\
2500.0)\end{array}$ & $\begin{array}{l}200.0 \\
(30.0- \\
2000.0)\end{array}$ & 0.047 & $\begin{array}{l}500.0 \\
(250.0- \\
2500.0)\end{array}$ & $\begin{array}{l}150.0 \\
(0.0- \\
200.0)\end{array}$ & $\begin{array}{l}< \\
0.001\end{array}$ \\
\hline $\begin{array}{l}\text { Intraoperative } \\
\text { erythrocyte } \\
\text { transfusion }\end{array}$ & $4(6.3)^{1}$ & $2(7.1)^{1}$ & $2(5.6)$ & 1.000 & $\begin{array}{l}4(12.1) \\
1\end{array}$ & 0 & 0.113 \\
\hline \multicolumn{8}{|l|}{ NE regimen } \\
\hline $\begin{array}{l}\text { NE dose on } \\
\text { surgery's start } \\
\left(\text { ug } \cdot \mathrm{kg}^{-1} \cdot \mathrm{min}^{-1}\right)\end{array}$ & $\begin{array}{l}0.05 \\
(0.00- \\
0.20)^{2}\end{array}$ & $\begin{array}{l}0.05 \\
(0.01- \\
0.20)^{1}\end{array}$ & $\begin{array}{l}0.05(0.00- \\
0.20)^{1}\end{array}$ & 0.472 & $\begin{array}{l}0.05 \\
(0.00- \\
0.19)^{2}\end{array}$ & $\begin{array}{l}0.05 \\
(0.00- \\
0.20)\end{array}$ & 0.692 \\
\hline
\end{tabular}




\begin{tabular}{|c|c|c|c|c|c|c|c|}
\hline \multirow[t]{3}{*}{ Variables } & \multirow[t]{3}{*}{$\begin{array}{l}\text { RAPD (n } \\
=64)\end{array}$} & \multicolumn{3}{|c|}{ Post-RAPD morbidity } & \multicolumn{3}{|c|}{$\begin{array}{l}\text { Intraoperative blood loss } \\
\text { (RAPD) }\end{array}$} \\
\hline & & \multirow{2}{*}{$\begin{array}{l}\text { RAPD } \\
\text { with } \\
\text { major } \\
\text { morbidity } \\
(C D \geq I I I, \\
n=28)\end{array}$} & \multirow{2}{*}{\multicolumn{2}{|c|}{$\begin{array}{l}\text { RAPD } \\
\text { without } \\
\text { major } \\
\text { morbidity } \\
(C D<1 I I, n \\
=36)\end{array}$}} & \multirow[t]{2}{*}{$\begin{array}{l}\text { High } \\
\left(\begin{array}{l}(\geq 0 \mathrm{ml} \\
25033) \\
\mathrm{n}=33)\end{array}\right.\end{array}$} & \multirow[t]{2}{*}{$\begin{array}{l}\text { Low } \\
(< \\
250 \mathrm{ml}, \\
\mathrm{n}=31)\end{array}$} & \multirow[t]{2}{*}{$P$} \\
\hline & & & & & & & \\
\hline $\begin{array}{l}\text { NE dose on } \\
\text { surgery's end } \\
\left(\mathrm{ug} \cdot \mathrm{kg}^{-1} \cdot \mathrm{min}^{-1}\right)\end{array}$ & $\begin{array}{l}0.06 \\
(0.00- \\
0.38)^{1}\end{array}$ & $\begin{array}{l}0.09 \\
(0.00- \\
0.22)^{1}\end{array}$ & $\begin{array}{l}0.04(0.00- \\
0.38)\end{array}$ & 0.726 & $\begin{array}{l}0.09 \\
(0.00- \\
0.38)^{1}\end{array}$ & $\begin{array}{l}0.04 \\
(0.00- \\
0.25)\end{array}$ & 0.518 \\
\hline $\begin{array}{l}\text { NE dose }>0.2 \\
\mathrm{ug} \cdot \mathrm{kg}^{-1} \cdot \mathrm{min}^{-1} \\
(\mathrm{~min})\end{array}$ & $\begin{array}{l}1(0-5) \\
1\end{array}$ & $1(0-5)^{1}$ & $1(0-3)$ & 0.471 & $\begin{array}{l}1(0-4) \\
1\end{array}$ & $1(0-5)$ & 0.374 \\
\hline $\begin{array}{l}\text { Time span NE dose } \\
>0.2 \mathrm{ug}^{\circ} \mathrm{kg}^{-1} \cdot \mathrm{min}^{-1} \\
(\mathrm{~min})\end{array}$ & $\begin{array}{l}4.1(0.0- \\
610.0)^{1}\end{array}$ & $\begin{array}{l}133.0 \\
\left(0.0^{-}\right. \\
610.0)^{1}\end{array}$ & $\begin{array}{l}22.5(0.0- \\
550.0)\end{array}$ & 0.262 & $\begin{array}{l}134.0 \\
(0.0- \\
600.0) \\
1\end{array}$ & $\begin{array}{l}15.0 \\
(0.0- \\
610.0)\end{array}$ & 0.283 \\
\hline $\begin{array}{l}\text { Operative time NE } \\
\text { dose }>\mathrm{ug} \cdot \mathrm{kg}^{-} \\
{ }^{1} \cdot \mathrm{min}^{-1}(\%)\end{array}$ & $\begin{array}{l}9.5(0.0- \\
96.2)^{1}\end{array}$ & $\begin{array}{l}20.4(0.0- \\
96.2)^{1}\end{array}$ & $\begin{array}{l}5.0(0.0- \\
9.1)\end{array}$ & 0.298 & $\begin{array}{l}20.8 \\
(0.0- \\
86.2)^{1}\end{array}$ & $\begin{array}{l}4.0(0.0- \\
96.2)\end{array}$ & 0.431 \\
\hline \multicolumn{8}{|l|}{$\begin{array}{l}\text { First arterial BGA } \\
\text { during surgery }\end{array}$} \\
\hline Blood pH & $\begin{array}{l}7.32 \pm \\
0.06^{7}\end{array}$ & $\begin{array}{l}7.34 \pm \\
0.06^{3}\end{array}$ & $\begin{array}{l}7.31 \pm 0.06 \\
4\end{array}$ & 0.135 & $\begin{array}{l}7.31 \pm \\
0.06^{3}\end{array}$ & $\begin{array}{l}7.33 \pm \\
0.06^{4}\end{array}$ & 0.361 \\
\hline $\begin{array}{l}\text { Partial } \mathrm{CO}_{2} \\
\text { pressure }(\mathrm{kPa})\end{array}$ & $\begin{array}{l}6.2 \\
(4.3- \\
24.2)^{7}\end{array}$ & $\begin{array}{l}6.1(4.3- \\
8.1)^{3}\end{array}$ & $\begin{array}{l}6.3(5.3- \\
24.2)^{4}\end{array}$ & 0.681 & $\begin{array}{l}6.3 \\
(4.3- \\
8.4)^{3}\end{array}$ & $\begin{array}{l}6.1 \\
(5.1- \\
24.2)^{4}\end{array}$ & 0.695 \\
\hline $\begin{array}{l}\text { Corresponding end- } \\
\text { tidal } \mathrm{CO}_{2}(\mathrm{kPa})\end{array}$ & $\begin{array}{l}5.0 \pm 0.6 \\
15\end{array}$ & $5.0 \pm 0.7^{5}$ & $5.0 \pm 0.5^{10}$ & 0.895 & $\begin{array}{l}5.0 \\
(3.9- \\
6.5)^{8}\end{array}$ & $\begin{array}{l}5.0 \\
(3.9- \\
6.6)^{7}\end{array}$ & 0.703 \\
\hline $\begin{array}{l}\text { Lactate level } \\
(\mathrm{mmol} / \mathrm{L})\end{array}$ & $\begin{array}{l}0.7 \\
(0.3- \\
1.9)^{7}\end{array}$ & $\begin{array}{l}0.8(0.3- \\
1.9)^{3}\end{array}$ & $\begin{array}{l}0.7(0.4- \\
1.3)^{4}\end{array}$ & 0.403 & $\begin{array}{l}0.8 \\
(0.3- \\
1.6)^{3}\end{array}$ & $\begin{array}{l}0.7 \\
(0.4- \\
1.9)^{4}\end{array}$ & 0.411 \\
\hline $\begin{array}{l}\text { Hemoglobin count } \\
(\mathrm{mmol} / \mathrm{L})\end{array}$ & $\begin{array}{l}7.8 \pm 0.8 \\
7\end{array}$ & $7.9 \pm 0.8^{3}$ & $7.8 \pm 0.9^{4}$ & 0.526 & $\begin{array}{l}7.7 \pm \\
0.9^{3}\end{array}$ & $\begin{array}{l}8.0 \pm \\
0.8^{4}\end{array}$ & 0.180 \\
\hline \multicolumn{8}{|l|}{$\begin{array}{l}\text { First arterial BGA } \\
\text { upon HDU/ICU } \\
\text { admission }\end{array}$} \\
\hline Blood pH & $\begin{array}{l}7.33 \pm \\
0.041\end{array}$ & $\begin{array}{l}7.32 \pm \\
0.04\end{array}$ & $\begin{array}{l}7.34 \pm 0.04 \\
1\end{array}$ & 0.017 & $\begin{array}{l}7.33 \pm \\
0.05^{1}\end{array}$ & $\begin{array}{l}7.33 \pm \\
0.04\end{array}$ & 0.588 \\
\hline
\end{tabular}




\begin{tabular}{|c|c|c|c|c|c|c|c|}
\hline \multirow[t]{3}{*}{ Variables } & \multirow[t]{3}{*}{$\begin{array}{l}\text { RAPD (n } \\
=64)\end{array}$} & \multicolumn{3}{|c|}{ Post-RAPD morbidity } & \multicolumn{3}{|c|}{$\begin{array}{l}\text { Intraoperative blood loss } \\
\text { (RAPD) }\end{array}$} \\
\hline & & \multirow{2}{*}{$\begin{array}{l}\text { RAPD } \\
\text { with } \\
\text { major } \\
\text { morbidity } \\
(C D \geq I I I, \\
n=28)\end{array}$} & \multirow{2}{*}{\multicolumn{2}{|c|}{$\begin{array}{l}\text { RAPD } \\
\text { without } \\
\text { major } \\
\text { morbidity } \\
(C D<\text { III, } n \\
=36)\end{array}$}} & \multirow[t]{2}{*}{$\begin{array}{l}\text { High } \\
\left(\begin{array}{l}250 \mathrm{ml}, \\
\mathrm{n}=33)^{\prime}\end{array}\right.\end{array}$} & \multirow[t]{2}{*}{$\begin{array}{l}\text { Low } \\
\begin{array}{l}(< \\
\mathrm{n}=31)\end{array}\end{array}$} & \multirow[t]{2}{*}{$P$} \\
\hline & & & & & & & \\
\hline $\begin{array}{l}\text { Partial } \mathrm{CO}_{2} \\
\text { pressure }(\mathrm{kPa})\end{array}$ & $\begin{array}{l}5.8 \\
(4.7- \\
20.1)^{1}\end{array}$ & $\begin{array}{l}5.8(4.8- \\
20.1)\end{array}$ & $\begin{array}{l}5.8(4.6- \\
10.7)^{1}\end{array}$ & 0.241 & $\begin{array}{l}5.8 \\
(4.7- \\
20.1)^{1}\end{array}$ & $\begin{array}{l}5.8(5.0- \\
10.7)\end{array}$ & 0.588 \\
\hline $\begin{array}{l}\text { Lactate level } \\
(\mathrm{mmol} / \mathrm{L})\end{array}$ & $\begin{array}{l}1.3 \\
(0.1- \\
6.3)^{1}\end{array}$ & $\begin{array}{l}1.7(0.5- \\
6.3)\end{array}$ & $\begin{array}{l}1.3(0.1- \\
6.3)^{1}\end{array}$ & 0.021 & $\begin{array}{l}1.6 \\
(0.5- \\
6.3)^{1}\end{array}$ & $\begin{array}{l}1.0 \\
(0.1- \\
3.7)\end{array}$ & 0.008 \\
\hline $\begin{array}{l}\text { Hemoglobin count } \\
(\mathrm{mmol} / \mathrm{L})\end{array}$ & $\begin{array}{l}7.8 \pm 0.9 \\
1\end{array}$ & $7.8 \pm 0.9$ & $7.8 \pm 0.9^{1}$ & 0.874 & $\begin{array}{l}7.5 \pm \\
0.9^{1}\end{array}$ & $\begin{array}{l}8.1 \pm \\
0.8\end{array}$ & 0.007 \\
\hline \multicolumn{8}{|l|}{$\begin{array}{l}\text { First arterial BGA } \\
\text { after } \geq 12 \text { hours } \\
\text { HDU/ICU } \\
\text { admission }\end{array}$} \\
\hline Blood pH & $\begin{array}{l}7.39 \\
(7.29- \\
7.48)^{4}\end{array}$ & $\begin{array}{l}7.37 \pm \\
0.041\end{array}$ & $\begin{array}{l}7.39 \pm 0.03 \\
3\end{array}$ & 0.016 & $\begin{array}{l}7.38 \pm \\
0.04^{3}\end{array}$ & $\begin{array}{l}7.40 \pm \\
0.041\end{array}$ & 0.131 \\
\hline $\begin{array}{l}\text { Partial } \mathrm{CO}_{2} \\
\text { pressure }(\mathrm{kPa})\end{array}$ & $\begin{array}{l}5.8(4.7- \\
7.0)^{4}\end{array}$ & $5.8 \pm 0.5^{1}$ & $5.6 \pm 0.5^{3}$ & 0.190 & $\begin{array}{l}5.8 \\
(4.8- \\
6.3)^{3}\end{array}$ & $\begin{array}{l}5.7(4.9- \\
7.0)^{1}\end{array}$ & 0.906 \\
\hline $\begin{array}{l}\text { Lactate level } \\
(\mathrm{mmol} / \mathrm{L})\end{array}$ & $\begin{array}{l}1.1 \\
(0.5- \\
2.9)^{4}\end{array}$ & $\begin{array}{l}1.4(0.6- \\
2.8)^{1}\end{array}$ & $\begin{array}{l}0.9(0.5- \\
2.9)^{3}\end{array}$ & 0.014 & $\begin{array}{l}1.2 \\
(0.7- \\
2.8)^{3}\end{array}$ & $\begin{array}{l}1.0 \\
(0.5- \\
2.9)^{1}\end{array}$ & 0.282 \\
\hline $\begin{array}{l}\text { Hemoglobin count } \\
(\mathrm{mmol} / \mathrm{L})\end{array}$ & $\begin{array}{l}7.5 \pm 0.9 \\
4\end{array}$ & $\begin{array}{l}7.7(5.2- \\
8.7)^{1}\end{array}$ & $\begin{array}{l}7.6(6.1- \\
9.5)^{3}\end{array}$ & 0.864 & $\begin{array}{l}7.1 \pm \\
0.9^{3}\end{array}$ & $\begin{array}{l}7.9 \pm \\
0.7^{1}\end{array}$ & $<$. \\
\hline \multicolumn{8}{|c|}{$\begin{array}{l}\text { Values are presented as number (proportion) or depending on normality distribution of cases as } \\
\text { mean } \pm \text { SD or median (interquartile range). } X^{n} \text { where } n \text { represents the number of missing cases. BGA, } \\
\text { Blood Gas Analysis; HDU, High Dependency Unit; ICU, Intensive Care Unit; NE, Norepinephrine; OPD, } \\
\text { Open Pancreatoduodenectomy; RAPD, Robot Assisted Pancreatoduodenectomy. }\end{array}$} \\
\hline
\end{tabular}


Table 5

Postoperative outcome after RAPD

\begin{tabular}{|c|c|c|c|c|c|c|c|}
\hline \multirow[t]{2}{*}{ Variable } & \multirow[t]{2}{*}{$\begin{array}{l}\text { RAPD } \\
(n=64)\end{array}$} & \multicolumn{3}{|c|}{ Post-RAPD morbidity } & \multicolumn{3}{|c|}{$\begin{array}{l}\text { Intraoperative (RAPD) } \\
\text { blood loss }\end{array}$} \\
\hline & & $\begin{array}{l}\text { RAPD with } \\
\text { major } \\
\text { morbidity } \\
\text { (CD } \geq \mathrm{III}, \mathrm{n}= \\
28)\end{array}$ & $\begin{array}{l}\text { RAPD } \\
\text { without } \\
\text { major } \\
\text { morbidity } \\
(C D<I I I, n= \\
\text { (C)) }\end{array}$ & $P$ & $\begin{array}{l}\text { High } \\
\left(\begin{array}{l}\geq \\
250 \mathrm{ml}, \\
\mathrm{n}=33)\end{array}\right.\end{array}$ & $\begin{array}{l}\text { Low } \\
(< \\
250 \mathrm{ml}, \\
\mathrm{n}=31)\end{array}$ & $P$ \\
\hline $\begin{array}{l}\text { Time to } \\
\text { detubation (min) }\end{array}$ & $\begin{array}{l}32.0 \\
(0.0- \\
931.0) \\
11\end{array}$ & $\begin{array}{l}49.5(0.0- \\
403.0)^{6}\end{array}$ & $\begin{array}{l}24.0(0.0- \\
931.0)^{5}\end{array}$ & 0.316 & $\begin{array}{l}55.0 \\
(0.0- \\
931.0) \\
8\end{array}$ & $\begin{array}{l}22.5 \\
(0.0- \\
185.0) \\
3\end{array}$ & 0.327 \\
\hline $\begin{array}{l}\text { Stay HDU/ICU } \\
\text { (hours) }\end{array}$ & $\begin{array}{l}11.5 \\
(4.0- \\
61.0)\end{array}$ & $\begin{array}{l}20.0(15.3- \\
97.4)\end{array}$ & $\begin{array}{l}19.1(14.6- \\
44.0)\end{array}$ & 0.140 & $\begin{array}{l}18.8 \\
(14.0- \\
97.4)\end{array}$ & $\begin{array}{l}19.7 \\
(14.6- \\
44.9)\end{array}$ & 0.122 \\
\hline $\begin{array}{l}\text { Prolonged } \\
\text { HDU/ICU } \\
\text { admission }\end{array}$ & $5(7.8)$ & $4(14.3)$ & $1(2.8)$ & 0.159 & $3(9.1)$ & $2(6.5)$ & 1.000 \\
\hline $\begin{array}{l}\text { Hospital stay } \\
\text { (days) }\end{array}$ & $\begin{array}{l}10.5 \\
(4.0- \\
61.0)\end{array}$ & $\begin{array}{l}19.0(5.0- \\
61.0)\end{array}$ & $\begin{array}{l}8.0(4.0- \\
37.0)\end{array}$ & $<0.001$ & $\begin{array}{l}17.0 \\
(5.0- \\
61.2)\end{array}$ & $\begin{array}{l}9.0 \\
(4.0- \\
48.0)\end{array}$ & 0.002 \\
\hline $\begin{array}{l}\text { Comprehensive } \\
\text { Complication } \\
\text { Index }\end{array}$ & $\begin{array}{l}32.7 \\
(0.0- \\
100.0)\end{array}$ & $\begin{array}{l}64.5(0.0- \\
100.0)\end{array}$ & $\begin{array}{l}21.8(0.0- \\
100.0)\end{array}$ & $<.001$ & $\begin{array}{l}51.5 \\
(12.2- \\
100.0)\end{array}$ & $\begin{array}{l}24.2 \\
(0.0- \\
99.9)\end{array}$ & $\dot{0} 001$ \\
\hline $\mathrm{CD} \geq$ Grade III & $\begin{array}{l}28 \\
(43.8)\end{array}$ & - & - & - & $\begin{array}{l}20 \\
(60.6)\end{array}$ & $\begin{array}{l}8 \\
(25.8)\end{array}$ & 0.006 \\
\hline $\begin{array}{l}\text { NRS } \\
\text { Postoperative } \\
\text { day } 1\end{array}$ & $\begin{array}{l}3(0-7) \\
8\end{array}$ & $3(0-7)^{1}$ & $2(0-7)^{7}$ & 0.248 & $\begin{array}{l}3(0- \\
7)^{3}\end{array}$ & $\begin{array}{l}2(0- \\
7)^{5}\end{array}$ & 0.255 \\
\hline $\begin{array}{l}\text { NRS } \\
\text { Postoperative } \\
\text { day } 3\end{array}$ & $\begin{array}{l}2(0-7) \\
9\end{array}$ & $2(0-7)^{3}$ & $2(0-4)^{6}$ & 0.071 & $\begin{array}{l}2(0- \\
7)^{4}\end{array}$ & $\begin{array}{l}2(0- \\
7)^{5}\end{array}$ & 0.562 \\
\hline $\begin{array}{l}\text { Values are presen } \\
\text { the number of mi } \\
\text { NRS, Numeric Rat } \\
\text { Pancreatoduoden }\end{array}$ & $\begin{array}{l}\text { as numl } \\
\text { g cases. } \\
\text { Scale; } 0 \\
\text { my. }\end{array}$ & $\begin{array}{l}\text { proportio } \\
\text { Clavien [ } \\
\text { Open Pan }\end{array}$ & $\begin{array}{l}\text { nedian (int } \\
\text { HDU, High } \\
\text { oduodenec }\end{array}$ & $\begin{array}{l}\text { tile ra } \\
\text { idenc } \\
\text { RAPL }\end{array}$ & $\begin{array}{l}X^{n} \text { y } \\
\text { it; ICU } \\
\text { bot As }\end{array}$ & $\begin{array}{l}\text { e n repr } \\
\text { ensive } \\
\text { ted }\end{array}$ & Unit; \\
\hline
\end{tabular}

\section{High vs. low intraoperative blood loss in RAPD}

Average intraoperative blood loss was $250 \mathrm{ml}$ in RAPD (Table 2), $\mathrm{n}=33$ RAPD procedures were characterized by high ( $\geq 250 \mathrm{ml}$ ) vs. 31 RAPD procedures by low $(<250 \mathrm{ml})$ intraoperative blood loss. Both operating room and operative time were longer in the RAPD group with high intraoperative blood 
loss (580.0 vs. 487.0 min., $p=0.001$ and 518.0 vs. 410.0 min., $p=0.001$, respectively, Table 4) compared to the RAPD group with low intraoperative blood loss. Average NE dose was higher at the end of surgery for the RAPD group with high intraoperative blood loss ( $0.09 \mathrm{vs.} 0.04 \mathrm{\mu g} \mathrm{kg}^{-1} \mathrm{~min}^{-1}$ in the RAPD group with low intraoperative blood loss). NE-dose trended to exceed $0.2 \mu \mathrm{g} \mathrm{kg}^{-1} \mathrm{~min}^{-1}$ more frequently in the RAPD group of high intraoperative blood loss (134.0 vs. $15.0 \mathrm{~min}, p=0.283$, and $20.8 \%$ vs. $4.0 \%$ of operative time in the RAPD group of low intraoperative blood loss, $p=0.431)$. Upon HDU/ICU admission, lactate levels were higher in the RAPD group of high intraoperative blood loss ( $1.6 \mathrm{vs} .1 .0 \mathrm{mmol} \mathrm{I}^{-1}$ in the RAPD group of low intraoperative blood loss, $p=0.008$ ). Length of hospital stay was doubled for the RAPD group of high intraoperative blood loss (16.0 vs. 8.0 days in the RAPD group of low intraoperative blood loss, $p=0.002$, Table 5 ). A higher rate of postoperative morbidity was observed in the RAPD group with high intraoperative blood loss (Comprehensive Complication Index of 51.5 vs. 24.2 in the RAPD group with low intraoperative blood loss, $p<0.001$ ). Besides, a higher rate of major postoperative morbidity ( $C D \geq$ III) was observed in the RAPD group of high intraoperative blood loss $(20 / 33,60.6 \%$ vs. $8 / 31,25.8 \%$ in the RAPD group of low intraoperative blood loss, $p=0.006$ ).

\section{Predictors of major morbidity after RAPD}

After univariate logistic regression analysis, anesthesia-related factors independently associated with major morbidity (CD $\geq \mathrm{III}$ ) after RAPD were a baseline medical history of hypertension (OR $3.51,95 \% \mathrm{Cl}$ $1.24-9.92, p=0.018$ ), colloid administration during surgery (OR 5.06, 95\% $\mathrm{Cl} 1.69-15.14, p=0.004$ ), lactate level upon $\mathrm{HDU} / \mathrm{ICU}$ admission (OR 2.47, 95\% Cl 1.27-4.82, $\mathrm{p}=0.008$ ) and lactate level after 12 hour HDU/ICU admission (OR 3.66, 95\% Cl 1.29-10.44, p = 0.015, Table 6). After backward stepwise regression, colloid administration during surgery and lactate level after 12 hour HDU/ICU admission remained independently associated with major morbidity after RAPD (OR 5.06, 95\% Cl 1.49-17.2, p= 0.009 and OR $3.18,95 \% \mathrm{Cl} 1.01-9.91, \mathrm{p}=0.047$, respectively). 
Table 6

Logistic regression analysis : anesthesia-related factors independently associated with major morbidity $(C D \geq I I)$ after RAPD

\begin{tabular}{|c|c|c|c|c|c|c|}
\hline \multirow[t]{2}{*}{ Variable } & \multicolumn{3}{|c|}{ Univariable analysis } & \multicolumn{3}{|c|}{$\begin{array}{l}\text { Backward stepwise } \\
\text { regression }\end{array}$} \\
\hline & OR & $95 \% \mathrm{Cl}$ & $P$ & OR & $\begin{array}{l}95 \% \\
\mathrm{Cl}\end{array}$ & $P$ \\
\hline Medical history of hypertension & 3.51 & $\begin{array}{l}1.24 \text { to } \\
9.92\end{array}$ & 0.018 & 3.28 & $\begin{array}{l}0.97 \\
\text { to } \\
11.13\end{array}$ & 0.057 \\
\hline Intraoperative colloid administration & 5.06 & $\begin{array}{l}1.69 \text { to } \\
15.14\end{array}$ & 0.004 & 5.06 & $\begin{array}{l}1.49 \\
\text { to } \\
17.20\end{array}$ & 0.009 \\
\hline Blood loss during surgery (ml) & 1.12 & $\begin{array}{l}1.00 \text { to } \\
1.26\end{array}$ & 0.058 & & & \\
\hline Operating room time (min) & 1.00 & $\begin{array}{l}1.00 \text { to } \\
1.01\end{array}$ & 0.599 & & & \\
\hline $\begin{array}{l}\text { Lactate level in first BGA after HDU/ICU } \\
\text { admisison }(\mathrm{mmol} / \mathrm{L})\end{array}$ & 2.47 & $\begin{array}{l}1.27 \text { to } \\
4.82\end{array}$ & 0.008 & & & \\
\hline $\begin{array}{l}\text { Lactate level in BGA after } \geq 12 \text { hour HDU/ICU } \\
\text { admisison (mmol/L) }\end{array}$ & 3.66 & $\begin{array}{l}1.29 \text { to } \\
10.44\end{array}$ & 0.015 & 3.18 & $\begin{array}{l}1.01 \\
\text { to } \\
9.91\end{array}$ & 0.047 \\
\hline
\end{tabular}

\section{Discussion}

After comparing perioperative anesthetic factors for RAPD and OPD, RAPD is characterized by higher demands of vasopressor support and higher intra- and postoperative $\mathrm{pCO}_{2}$ and $\mathrm{Hb}$-levels. Although net intraoperative fluid balance and vasopressor demands are higher in RAPD, levels of colloid and erythrocyte transfusion are lower for RAPD compared to OPD. Rates of major postoperative morbidity $(C D \geq I I I)$ were similar for the surgical approaches. Within patients who developed major morbidity (CD $\geq$ III) after RAPD, vasopressor demands and necessity of colloid administration tended to be higher. The need for intraoperative colloid administration and increased postoperative lactate levels were independently associated with major morbidity (CD $\geq$ III) after RAPD.

Comparing outcomes, the surgical modality itself influences the development of major morbidity less than patient-related variables. We report a small fraction of patients marked ASA class III and above $(11 / 64,17.2 \%$ in RAPD and $13 / 62,22.4 \%$ in OPD), compared to earlier studies reporting percentages up to 43.1 and $82.4 \%[14,15]$. This discrepancy might suggest an underrating of ASA grading, in contrast with the 2017 strengthened ASA classifications [16]. ASA scores in our series are however in concordance with 
recent findings of van Roessel et al., reporting 21.8\% ASA III patients in a cohort of $n=3341$ pancreatoduodenectomy and distal pancreatectomy procedures, using Dutch Nationwide Pancreatic Cancer Audit data $[17,18]$. Van Roessel et al. predict worse outcome after pancreatoduodenectomy in $A S A \geq$ III patients (OR $0.59,95 \% \mathrm{Cl} 0.44-0.80$, for achieving optimal outcome after pancreatic surgery). In our study ASA class itself was not an individual predictor for postoperative major morbidity (CD $\geq$ III) where baseline hypertension was (OR 3.51, 95\% $\mathrm{Cl} 1.24-9.92)$. This finding, compared with higher vasopressor demands in RAPD, implies as association between baseline cardiovascular condition and postoperative outcome after RAPD. However, a medical history of hypertension might comprise several baseline conditional factors with itself a possible influence on postoperative morbidity (e.g. increased $\mathrm{BMI}$, vascular remodeling or pre-existing renal insufficiency).

A higher need for intraoperative vasopressor administration in RAPD could be explained by differences in patient positioning (reversed-Trendelenburg in RAPD vs. supine in OPD) as well as exposure to pneumoperitoneum, affecting cardiac afterload and cardiac output [7-9]. Higher demand for vasopressor administration in RAPD was not necessarily reflected by worse baseline physical condition. Although OPD patients more often received neoadjuvant chemotherapy and baseline hemoglobin levels were lower, no differences in baseline medical condition could be demonstrated between both surgical approaches. Although the intraoperative use of vasopressors was evident, we feel supported by recently published data that routinely insertion of a central venous catheter is not mandatory in RAPD or OPD patients [19, 20].

The 2018 RELIEF Study reported on postoperative outcome after distinct intraoperative fluid strategies during major abdominal surgery, differentiating between an either restrictive (median crystalloid + colloid 2177) or liberal (median crystalloid + colloid $3500 \mathrm{ml}$ ) net intraoperative fluid balance [5]. Whereas no differences were observed in general postoperative outcome between both fluid approaches, a liberal intraoperative fluid strategy was associated with lower rates of postoperative kidney failure $(17 / 1439$, $5.0 \%$ for liberal vs. $124 / 1443,8.6 \%$ for restrictive, $p<0.001)$. In comparison, we report a median intraoperative fluid balance of $2800 \mathrm{ml}$ and a 9/64 (14.5\%) rate of postoperative acute kidney injury in RAPD patients. Bannone et al. observed an increased rate of post pancreatoduodenectomy pancreatitis in patients exposed to a near-zero net perioperative fluid regime, suggesting a more restrictive perioperative fluid balance to be associated with an increased risk of postoperative pancreatic fistula [21]. On the contrary, the 2019 meta-analysis by Garland et al. reported an OR of 0.54 ( $95 \% \mathrm{Cl} 0.31-0.94)$ for major morbidity post pancreatoduodenectomy surgery after following a more restrictive intraoperative fluid strategy [22]. The optimal intraoperative fluid regime in pancreatoduodenectomy remains point of debate and prospective research should extrapolate this topic to minimally-invasive vs. conventional pancreatoduodenectomy surgery.

We observed an association between the intraoperative administration of colloids and development of major morbidity after RAPD (OR 5.06, 95\% Cl 1.96-15.14, $\mathrm{p}=0.009$ ). This finding is in accordance with Simões, reporting an OR of $1.86(95 \% \mathrm{Cl} 1.03-4307)$ for development of major postoperative morbidity after the intraoperative administration of colloids $(n=308$ elective surgeries for abdominal malignancies, 
including $\mathrm{n}=22$ pancreatic surgical procedures) [23]. In our RAPD series of low intraoperative blood loss, $8 / 31$ (25.8\%) of patients vs. $25 / 33(75.8 \%)$ of patients in the RAPD group of high intraoperative blood loss intraoperative received colloids. It is important to consider which patient category requires intraoperative colloid transfusion. Since colloid administration is part of therapy for major blood loss in our center's protocol, the association of intraoperative colloid administration and development of major postoperative morbidity $(C D \geq I I I)$ does not necessarily have to reflect a direct causative effect.

Over the perioperative course, $\mathrm{pH}$ values were higher in RAPD compared to OPD (7.35 vs. 7.32 on beginning of surgery, $p=0.021$ and 7.35 vs. 7.33 upon HDU/ICU arrival, $p=0.013$ ). These differences in $\mathrm{pH}$ levels do not reflect clinical relevance and moderately higher perioperative $\mathrm{pH}$ levels in RAPD can well be explained by exposure to pneumoperitoneum and (retroperitoneal) absorption of $\mathrm{CO}_{2}$. In our series a higher lactate level after a minimum admission of 12 hours on HDU/ICU was associated with major morbidity after RAPD (OR 3.18, 95\% Cl 1.01-9.91, $\mathrm{p}=0.047)$. This is in accordance with De Schryver et al., reporting an OR of $3.58(95 \% \mathrm{Cl} 1.22-10.18, \mathrm{p}=0.020)$ for 6 -hour post pancreatic (laparotomic) surgery hyperlactatemia and development of postoperative pancreatic fistula [24]. Average postoperative pain scores during the first postoperative day were higher in RAPD compared to OPD. The reported first postoperative day NRS of 3 in RAPD compared to a NRS of 1 in OPD is of limited clinical relevance and therefore not attributable to major morbidity. Besides, this moderate difference can well be explained by the routine application of additional epidural analgesia in OPD, in accordance with previously reported studies on additional epidural analgesia during pancreatoduodenectomy [25].

Our study comprises several limitations. First the retrospective single-center study design covering a relatively high, but still limited number of procedures. Due to the limited number of surgeons, the surgical approach was very standardized. This is in contrast to the perioperative anesthetic care, which was provided by $n=39$ different consultant anesthesiologists who followed available protocols with different levels of adherence.

\section{Conclusions}

Specific differences exist in perioperative anesthesia-related factors between RAPD and OPD. RAPD is associated with higher levels of vasopressor drug administration as well as higher net perioperative fluid balance. Besides, levels of colloid and erythrocyte transfusion are lower for RAPD compared to OPD. Baseline hypertension, perioperative colloid administration and increased lactate levels after surgery were associated with higher rates of major morbidity $(C D \geq I I I)$ after RAPD. A more restrictive intraoperative fluid regime has previously been shown to increase postoperative (nephrogenic) morbidity, present evidence is however contradictory. Current data is insufficient to make specific recommendations on perioperative anesthetic guidance in RAPD. However, intraoperative hemodynamics including fluid strategy might influence postoperative morbidity and should be the focus of future prospective research.

\section{Abbreviations}




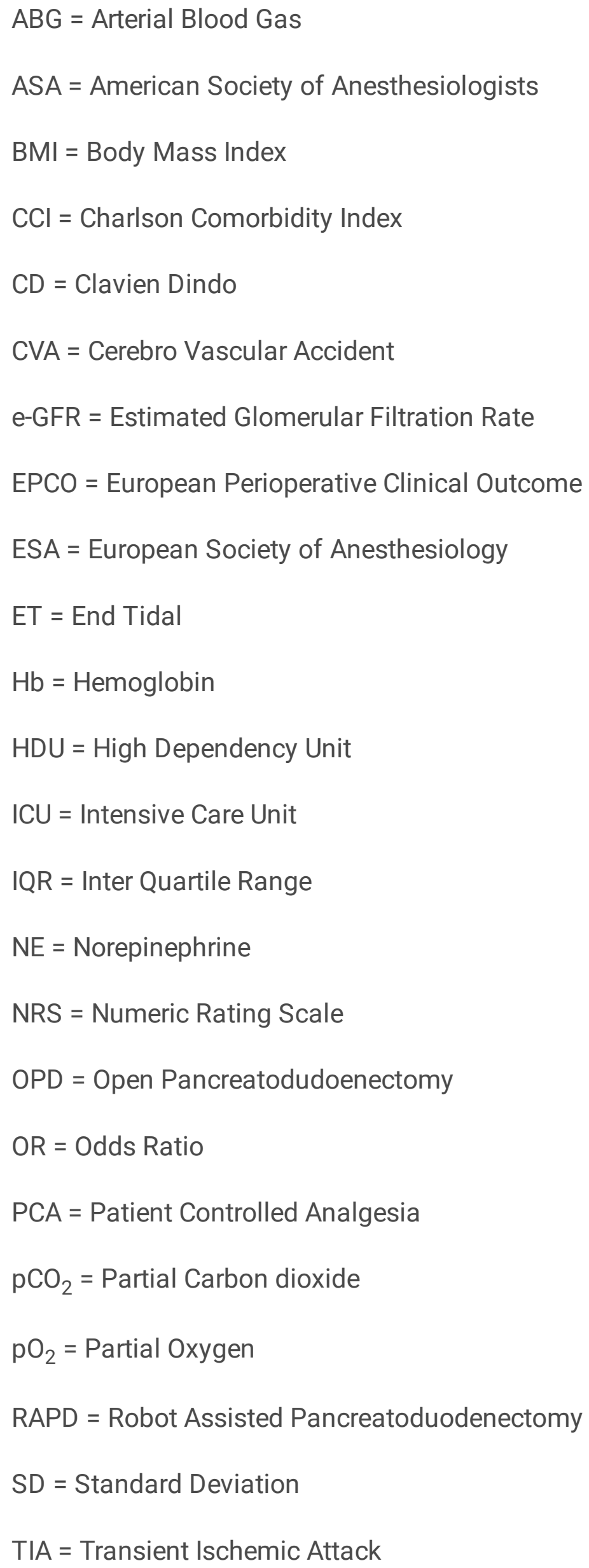




\section{Declarations}

\section{Ethics approval and consent to participate}

The local Medical Ethics Committee approved the study with waiver for informed patient consent with reference MEC-2019-0090 (Medical Ethics Committee, Erasmus MC University Hospital, Rotterdam, the Netherlands).

\section{Consent for publication}

Not applicable

\section{Availability of data and materials}

The datasets used and analysed during the current study are available from the corresponding author on reasonable request.

\section{Competing interests}

The authors declare that they have no competing interests.

\section{Funding}

Funding was provided solely from institutional and/or departmental sources.

\section{Authors' contributions}

AE was responsible for data acquisition, methodology, formal analysis and visualization of data. Conceptualization was performed by BGK and MK. Writing of the original draft was conducted by AE. BGK, MMV and MK reviewed and edited the manuscript. The final manuscript was read and approved by all authors.

\section{Acknowledgements}

The authors thank Khe T.C.K. Tran, MD, consultant surgeon (department of Surgery, Division of Hepatobiliary, Pancreatic- and Transplant Surgery at Erasmus University MC, Rotterdam, the Netherlands) for her help in implementing the robot-assisted pancreatoduodenectomy in our university hospital. We also acknowledge Casper H.J. van Eijck, MD, PhD, professor and consultant surgeon (department of Surgery, Division of Hepatobiliary, Pancreatic- and Transplant Surgery at Erasmus University MC, Rotterdam, the Netherlands) for leading and coordinating the pancreatic surgical team in our university hospital.

\section{References}


1. Giulianotti PC, Coratti A, Angelini M, Sbrana F, Secconi S, Balestracci T et al. Robotics in general surgery: personal experience in a large community hospital. Arch Surg. 2003 Jul; 138(7): 777 - 84. https://doi.10.001/archsurg.138.7.777.

2. Chen S, Chen JZ, Zhan Q, Deng XX, Shen BY, Peng CH et al. Robot-assisted laparoscopic versus open pancreaticoduodenectomy: a prospective, matched, mid-term follow-up study. Surg Endosc. 2015 Dec; 29 (12): 3698 - 711. https://doi.10.1007/s00464-015-4140-y.

3. Boggi U, Napoli N, Costa F, Kauffmann EF, Menonna F, lacopi, S et al. Robotic-assisted pancreatic resections. World J Surg. 2016 Oct; 40(10): 2497 - 506. https://doi.10.1007/s00268-016-3565-3.

4. Wang SE, Shyr BU, Chen SC, Shyr YM. Comparison between robotic and open pancreaticoduodenectomy with modified Blumgart pancreaticojejunostomy: a propensity scorematched study. Surgery. 2018 Dec; 164(6): 1162-7. https://doi.10.1016/j.surg.2018.06.031.

5. Myles PS, Bellomo R, Corcoran T, Forbes A, Peyton P, Story D et al. Restrictive versus liberal fluid therapy for major abdominal surgery. N Engl J Med. 2018 Jun; 378(24): 2263-74. https://doi.10.1056/NEJMoa1801601.

6. Grant F, Brennan MF, Allen PJ, DeMatteo RP, Kingham P, D'Angelica M et al. Prospective randomized controlled trial of liberal vs restricted perioperative fluid management in patients undergoing pancreatectomy. Ann Surg. 2016 Oct; 264(4): 591-8. https://doi.10.1097/SLA.0000000000001846.

7. Odeberg S, Ljungqvist O, Svenberg T, Gannedahl P, Bäckdahl M, von Rosen A et al. Hemodynamic effects of pneumoperitoneum and the influence of posture during anesthesia for laparoscopic surgery. Acta Anesthesiol Scand. 1994 Apr; 38(3): 276 - 83. https://doi.10.1111/j.13996576.1994.tb03889.x.

8. He H, Gruartmoner G, Ince Y, van Berge Henegouwen M, Gisbertz SS, Geerts BF et al. Effect of pneumoperitoneum and steep reverse-Trendelenburg position on mean systemic filling pressure, venous return, and microcirculation during esophagectomy. J Thorac Dis. 2018 Jun; 10(6): 3399 408. https://doi.10.21037/jtd.2018.05.169.

9. Atkinson TM, Giraud GD, Togioka BM, Jones DB, Cigarroa JE. Cardiovascular and ventilatory consequences of laparoscopic surgery. Circulation. 2017 Feb; 135(7): 700 - 10. https://doi.10.1161/CIRCULATIONAHA.116.023262.

10. Charlson ME, Pompei P, Ales KL, MacKenzie CR. A new method of classifying prognostic comorbidity in longitudinal studies: development and validation. J Chronic Dis. 1987; 40(5): $373-83$. https://doi.10.1016/0021-9681(87)90171-8.

11. Dindo D, Demartines $N$, Clavien PA. Classification of surgical complications: a new proposal with evaluation in a cohort of 6336 patients and results of a survey. Ann Surg. 2004 Aug; 240(2): 205 13. https://doi.10.1097/01.sla.0000133083.54934.e.

12. Slankamenac K, Graf R, Barkun J, Puhan MA, Clavien PA. The comprehensive complication index: a novel continuous scale to measure surgical morbidity. Ann Surg. 2013 Jul; 258(1): 1-7. https://doi.10.1097/SLA.0b013e318296c732. 
13. Jammer I, Wickboldt N, Sander M, Smith A, Schultz MJ, Paolo P et al. Standards for definitions and use of outcome measures for clinical effectiveness research in perioperative medicine: European Perioperative Clinical Outcome (EPCO) definitions: a statement from the ESA-ESICM joint taskforce on perioperative outcome measures. Eur J Anesthesiol. 2015 Feb; 32 (2): 88-105. https://doi.10.1097/EJA.0000000000000118.

14. Kim JH, Gonzalez-Heredia R, Daskalaki D, Rashdan M, Masrur M, Giulianotti PC. Totally replaced right hepatic artery in pancreaticoduodenectomy: is this anatomical condition a contraindication to minimally invasive surgery? HPB (Oxford). 2016 Jul; 18(7): 580-5.

https://doi.10.1016/j.hpb.2016.04.009.

15. Jung JP, Zenati MS, Dhir M, Zureikat AM, Zeh HJ, Simmons RL et al. Use of video review to investigate technical factors that may be associated with delayed gastric emptying after pancreaticoduodenectomy. JAMA Surg. 2018 Oct; 153(10): 918 - 27. https://doi.10.1001/jamasurg.2018.2089.

16. Hurwitz EE, Simon M, Vinta SR, Zehm CF, Shabot SM Minhajuddin A et al. Adding examples to the ASA-physical status classification improves correct assignment to patients. Anesthesiology. 2017 Apr; 126(4): 614 - 22. https://doi.10.1097/ALN.0000000000001541.

17. Van Roessel S, Mackay TM, Van Dieren S, Van der Schelling GP, Nieuwenhuijs VB, Bosscha K et al. Textbook outcome: nationwide analysis of a novel quality measure in pancreatic surgery. Ann Surg. 2020 Jan; 271(1): 155 - 62. https://doi.10.1097/SLA0000000000003451.

18. Bengt van Rijssen L, Groot Koerkamp B, Zwart MJ, Bonsing BA, Bosscha K, Van Dam RM et al. Nationwide prospective audit of pancreatic surgery: design, accuracy, and outcomes of the Dutch Pancreatic Cancer Audit. HPB (Oxford). 2017 Oct; 19(10): 919 - 26. https://doi.10.1016/j.hpb.2017.06.010.

19. Lewis T, Merchan C, Altshuler D, Papadopoulos J. Safety of the peripheral administration of vasopressor agents. J Intensive Care Med. 2019 Jan; 34(1): 26-33. https://doi.10.1177/0885066616686035.

20. Tian DH, Smyth C, Keijzers G, Mackdonald SP, Peake S, Udy A et al. Safety of peripheral administration of vasopressor medications: a systematic review. Emerg M Australas. 2020 Apr; 32(2): 220-7. https://doi.10.1111/1742-6723.13406.

21. Bannone E, Andrianello S, Marchegiani G, Masini G, Malleo G, Bassi C et al. Postoperative acute pancreatitis following pancreaticoduodenectomy: a determinant of fistula postentially driven by the intraoperative fluid management. Ann Surg. 2018 Nov; 268(5): 815 - 22.

https://doi.10.1097/SLA.0000000000002900.

22. Garland ML, Mace HS, MacCormick AD, McCluskey SA, Lightfoot N. Restrictive versus liberal fluid regimens in patients undergoing pancreaticoduodenectomy: a systematic review and meta-analysis. J Gastrointest Surg. 2019 Jun; 23(6): 1250-65. https://doi.10.1007/s11605-018-04089-6.

23. Simões CM, Carmona MJC, Hajjar LA, Vincent JL, Landoni G, Belletti A. Predictors of major complications after elective abdominal surgery in cancer patients. BMC Anesthesiol. 2018 May; 
18(1): 49. https://doi.10.1186/s12871-018-0516-6.

24. De Schryver N, Wittebole X, Hubert C, Gigot JF, Laterre PF, Castanares-Zapatero D. Early hyperlactatemia predicts pancreatic fistula after surgery. BMC Anesthesiol. $2015 \mathrm{Jul} ; 28(15): 109$. https://doi.10.1186/s12871-015-0093-x.

25. Groen JV, Khawar AAJ, Bauer PA, Bonsing BA, Martini CH, Mungroop TH et al. Meta-analysis of epidural analgesia in patients undergoing pancreatoduodenectomy. BJS Open. 2019 Apr; 29(5): 559-571. https://doi.10.1002/bjs5.50171. 